\title{
Analysis of a trial-spacing effect with relatively long intertrial intervals
}

\author{
Ceyhun Sunsay and Mark E. Bouton \\ University of Vermont, Burlington, Vermont
}

\begin{abstract}
In three experiments with rat subjects, we examined the effects of trial spacing in appetitive conditioning. Previous research in this preparation suggests that self-generated priming of the conditional stimulus (CS) and/ or unconditional stimulus (US) in short-term memory is a cause of the trial-spacing effect that occurs with intertrial intervals (ITIs) of less than $240 \mathrm{sec}$. Experiment 1 nonetheless showed that a trial-spacing effect still occurs when ITIs are increased beyond $240 \mathrm{sec}$, and that the effect of ITI over 60-1,920 sec on conditioned responding is best described as a linear function. In Experiment 2, some subjects were removed from the context during the ITIs, preventing extinction of the context. Removal abolished the advantage of the long ITI, suggesting the importance of exposure to the context during the long ITI. Experiment 3 still produced a trial-spacing effect in a within-subjects design that controlled for the level of context conditioning and reinforcement rate in the absence of the CS. Overall, the results are most consistent with the idea that adding time to the ITI above $240 \mathrm{sec}$ facilitates conditioning by extinguishing context-CS associations - and possibly context-US associations - that otherwise interfere with CS-US learning through retrieval-generated priming (see, e.g., Wagner, 1981).
\end{abstract}

In Pavlovian conditioning, animals respond to a conditional stimulus (CS) after it has been paired with a biologically significant unconditional stimulus (US). One variable that modulates the strength of conditioning is the amount of time that elapses between successive CS-US pairings or trials. Generally speaking, the acquisition of conditioned responding proceeds more quickly when the conditioning trials are spaced in time; learning is positively related to the interval of time between trials. This trial-spacing effect has been documented in several conditioning preparations, including appetitive conditioning (see, e.g., Bouton \& Sunsay, 2003; Holland, 2000; Lattal, 1999), autoshaping (e.g., Gibbon, Baldock, Locurto, Gold, \& Terrace, 1977; Jenkins, Barnes, \& Barrera, 1981; Kaplan, 1984; Papini \& Brewer, 1994; Terrace, Gibbon, Farrell, \& Baldock, 1975), fear conditioning (Barela, 1999; Ewing, Larew, \& Wagner, 1985; Yeo, 1976; Yin, Barnet, \& Miller, 1994), eyelid conditioning (e.g., Salafia, Mis, Terry, Bartosiak, \& Daston, 1973; Spence \& Norris, 1950), and taste aversion learning (Domjan, 1980) in species ranging from the fruit fly (Drosophila melanogaster; Phelan, Rodd, Byers, \& Rosellini, 1998) and Hermissenda (Rogers, Talk, \& Matzel, 1994) to humans (e.g., Glenberg, 1976, 1979; Melton, 1970).

There are a number of explanations of the trial-spacing effect. One set of explanations emphasizes the role of contextual cues that are always present along with the CS during conditioning. These cues gain some associative strength along with the CS on each conditioning trial, since they are also present at the time of the US delivery. However, they can then lose some associative strength during the intertrial interval (ITI) when the US is withheld, and some extinction can occur. Context extinction then allows less blocking of the CS by the context: The CS can now gain more associative strength when the CScontext compound is next paired with the US. A number of findings are consistent with the view that the CS and the context compete for conditioning (Durlach, 1984; Grau \& Rescorla, 1984; Rescorla \& Durlach, 1987; Rescorla, Durlach, \& Grau, 1985; see Durlach, 1989, and Randich \& Ross, 1985, for reviews). According to models such as that of Rescorla-Wagner (see, e.g., Rescorla \& Wagner, 1972), the context blocks learning of the CS-US association. According to the comparator hypothesis (Miller \& Matzel, 1988; Miller \& Schachtman, 1985), the CS and the context compete at the level of performance rather than learning: Responding to a CS is determined by the relative conditioned strengths of the CS and its comparator stimulus (the context). High contextual conditioning resulting from massed conditioning trials causes the context to win the comparison (see Yin et al., 1994).

Wagner's sometimes opponent process model, or SOP (Wagner, 1981), suggests a blocking role for context-US associations like that envisioned by the Rescorla-Wagner model. According to SOP, stimuli are represented by hypothetical memory nodes that consist of a finite number of elements. When a stimulus is presented, its nodal elements are activated to a primary memory state called $A 1$. The elements remain active in $\mathrm{A} 1$ for a brief time and then decay to a less active memory state called $A 2$. Finally, elements lose their activation as they decay and return to

C.Sunsay, csunsay@indiana.edu 
the inactive state. The elements remain inactive until the stimulus is presented again. An excitatory association between the CS and the US develops when elements of both stimuli are active in the A1 state at the same time. As the excitatory association develops, the CS starts to prime the representation of the US into A2 state. This is how memory retrieval works; as a result of conditioning, the CS comes to activate the US memory to the A2 state. Putting the representation of a stimulus (e.g., a US) into A2 by presenting a stimulus with which it is associated (e.g., CS) is called retrieval-generated priming. During conditioning with a massed-trial procedure, the context might be associated with the US and prime the US representation to the A2 state, causing blocking. Spaced trials once again permit extinction of the context, and, therefore, less retrievalgenerated priming. Interestingly, SOP gives a similar role to context-CS associations that might be acquired during conditioning. With short ITIs, the animal also forms an association between the context and the CS. The context would then cause retrieval-generated priming of the CS node into A2, reducing its ability to become conditioned. Like the context-US association, a context-CS association would also extinguish to some extent during longer ITIs.

Another set of conditioning theories gives the context a different role. These models do not view learning as a process of building an association between a CS and a US. Rather, the animals estimate the rate of reinforcement (Gallistel \& Gibbon, 2000) in the CS as well as in the background (e.g., context) and make a decision to respond when the CS has a higher reinforcement rate than the background. In time-accumulation models (Gallistel \& Gibbon, 2000; Gibbon \& Balsam, 1981), it is assumed that organisms can learn and store durations of events, such as trials and ITIs, with the help of the internal clock and accumulator. Not only can animals estimate when a reinforcer is due and thus start or stop responding, but they can also evaluate the rate of the reinforcer in the CS or trial (T) and in the ITI (I). The comparison of the two reinforcement rates $\left(n_{\mathrm{T}} / n_{\mathrm{I}}\right)$ - or simply the comparison of the two intervals $(\mathrm{I} / \mathrm{T})$ - determines the rate of conditioning. When the ratio exceeds a certain threshold, the animal makes a decision to respond. If the ratio is large, the CR emerges quickly. In other words, the larger the ratio, the faster the conditioning.

According to these models, the trial-spacing effect occurs because the I/T ratio is larger when trials are spaced rather than massed. An important implication is that trial spacing is not an absolute effect. Massed and spaced trials might support equal rates of conditioning if the trial duration in the spaced condition is longer than that in the massed condition in such a way that their I/T ratios would be equal. That is, despite actual differences in trial and ITI durations, the rate of conditioning would be equal if $\mathrm{I} / \mathrm{T}$ ratios are equal. This is called timescale invariance, and it has received empirical support in the autoshaping preparation, in which pigeons peck illuminated keylights associated with food (see, e.g., Gibbon et al., 1977). However, some limitations have also been reported in other conditioning preparations (e.g., Bouton \& Sunsay, 2003; Holland, 2000; Kirkpatrick \& Church, 2000; Lattal, 1999; Sunsay, Stetson, \& Bouton, 2004).
The present experiments grew out of recent work in this laboratory on the trial-spacing effect (Bouton \& Sunsay, 2003; Sunsay et al., 2004). Bouton and Sunsay set out to test the time accumulation models' account of conditioning (Gallistel \& Gibbon, 2000; Gibbon \& Balsam, 1981). Such models predict an equal rate of conditioning in groups that receive an equal I/T ratio no matter how trials are presented. The Bouton and Sunsay experiments involved a partial reinforcement (PRF) procedure. In a PRF procedure, some presentations of the CS are not followed by the US. In contrast to trial-based explanations of conditioning, time accumulation models do not assume a decrement in associative strength as a result of the nonreinforced trials. Instead, they merely add accumulated trial time (T) to the I/T ratio. Nonetheless, groups were found to differ in their conditioning according to the number of nonreinforced trials, despite equal I/T ratios (Bouton \& Sunsay, 2003, Experiment 3 ). This finding clearly violates the time accumulation mechanism. In addition, groups differed depending on when in time the groups received a nonreinforced trial with respect to the next reinforced trial (Bouton \& Sunsay, 2003, Experiments 3 and 4). When the nonreinforced and reinforced trials were separated by only $60 \mathrm{sec}$, there was less conditioning than when there was a longer temporal gap $(240 \mathrm{sec})$. The groups differed despite the fact that they had equal I/T ratios.

Bouton and Sunsay (2003) suggested that these results were consistent with another mechanism suggested by SOP. When trials are massed, the CS and/or US can briefly prime itself into the A1 state (through self-generated priming) on trial $n$ and then decay to $\mathrm{A} 2$ by trial $n+1$. As described above, this reduces conditioning. As long as trials are massed sufficiently, the node may be in A2 when trial $n+1$ occurs. When trials are spaced further, the elements have time to fully decay to the inactive state, and, by the time of the next trial, they can be activated to the A1 state. Consistent with this analysis, Sunsay et al. (2004) further found that the CS on a nonreinforced priming trial had to be the same as the CS on the target trial, suggesting a CS self-generated priming mechanism. When trials were preceded by either a CS presentation, a US presentation, or a CS-US pairing, the resulting priming effect was essentially over when the interval between the prime and the target trial was $240 \mathrm{sec}$ (see also Bouton \& Sunsay, 2003). The results thus suggest that self-generated priming may play a role in massed-trial procedures in which the interval between trials is less than $240 \mathrm{sec}$. Nonetheless, there was preliminary evidence that the trial-spacing effect might occur with ITIs longer than $240 \mathrm{sec}$ (e.g., Bouton \& Sunsay, 2003). Therefore, selfgenerated priming may not be the only explanation of the trial-spacing effect in this preparation.

The present experiments were therefore aimed at further investigating the trial-spacing effect as it occurs in the appetitive conditioning preparation used by Bouton and Sunsay (2003) and Sunsay et al. (2004), with ITIs greater than $240 \mathrm{sec}$. The first experiment was designed to test whether a trial-spacing effect still occurs when trials were spaced beyond this value. After confirming that it does, Experiments 2 and 3 were designed to test the mechanisms behind the trialspacing effect that occurs with these relatively long ISIs. 


\section{EXPERIMENT 1}

The first experiment compared conditioning in groups that received tone-food pairings separated by ITIs of 60,120 , $180,240,480,960,1,440$, and 1,920 sec. As described previously, if the trial-spacing effect occurs beyond the estimated boundary of self-generated priming $(240 \mathrm{sec})$, then another mechanism or mechanisms would be implicated in accounting for the trial-spacing effect. The experiment included a substantial range of ITIs so that we could evaluate the quantitative relationship between the ITI and the level of responding that develops during conditioning in this preparation.

\section{Method}

\section{Subjects}

The subjects were 64 female Wistar rats obtained from Charles River, Inc., in St. Constance, Quebec. They were approximately 85-90 days of age at the start of the experiment. They were individually housed in suspended stainless-steel cages in a room maintained on a 12:12 h light:dark cycle. The rats were food deprived to $80 \%$ of their free-feeding weights throughout the experiment.

\section{Apparatus}

Two sets of Skinner boxes were housed in sound attenuation chambers located in separate rooms of the laboratory. Although the different sets are designed to function as different contexts, they were not used in that capacity in Experiment 1 . The boxes from both sets measured $31.75 \times 24.13 \times 29.21 \mathrm{~cm}(1 \times \mathrm{w} \times \mathrm{h})$; the side walls and ceiling were made of clear acrylic plastic, whereas the front and rear walls were made of brushed aluminum. Recessed $5.08 \times 5.08 \mathrm{~cm}$ food cups were centered in the front wall at about the level of the floor. These cups had infrared photo beams positioned approximately $1.2 \mathrm{~cm}$ behind the plane of the wall and $1.2 \mathrm{~cm}$ above the bottom of the cup. In one set of four boxes, the floor was composed of stainless steel grids ( $0.48 \mathrm{~cm}$ in diameter), spaced $3.18 \mathrm{~cm}$ center-to-center. One side wall had black horizontal stripes that were $3.81 \mathrm{~cm}$ wide and $3.81 \mathrm{~cm}$ apart. The ceiling had similarly spaced stripes oriented in the same direction. A distinctive odor was created by leaving a cup of about $10 \mathrm{ml}$ of a $2 \%$ McCormick anise extract solution in a dish outside the front wall of the Skinner box. In the other set of four boxes, the floor was made of alternating stainless steel grids of different diameters $(0.48 \mathrm{~cm}$ and $1.27 \mathrm{~cm}$ ) that were separated by $1.59 \mathrm{~cm}$ center-to-center. One side wall and the ceiling were covered with rows of dark dots (1.9-cm diameter), with the adjacent dots separated by about $1.27 \mathrm{~cm}$. A distinctive odor was created by leaving a cup of about $10 \mathrm{ml}$ of a $4 \%$ McCormick coconut extract solution in a dish outside the front wall of the Skinner box.

The CS was a $30-\mathrm{sec} 3,000-\mathrm{Hz}$ tone $(80 \mathrm{dBA})$ that was delivered through a $7.6-\mathrm{cm}$ speaker mounted to the ceiling of the sound attenuation chamber (background noise was maintained at $60 \mathrm{dBA}$ ). The US was two 45-mg food pellets (Improved Formula A, Noyes, Lancaster, NH) that were delivered at the offset of the CS.

\section{Procedure}

The experiment began with an initial 20-min magazine training session in which each rat was put in a box and given $31-34$ pellets to retrieve from the food cups.

The rats were then randomly assigned to eight groups $(n=8)$, with the restriction that the boxes were fully balanced. Beginning on the day following magazine training, all groups then received 16 daily sessions in which there were four reinforced trials, spaced by one of eight fixed ITI values: 60, 120, 180, 240, 480, 960, 1,440, and $1,920 \mathrm{sec}$. In each group, the rats were removed from the conditioning chambers within a minute after the last conditioning trial. After the final session, the groups were given 2 days of context exposure that was designed to extinguish contextual conditioning and thus equalize the groups' responding in the preperiod. The length of these context extinction sessions was 47 min (the mean length of the con- ditioning sessions of all groups). The next day, each group received four nonreinforced CS presentations that were spaced by the ITI that they had received during the conditioning phase.

The computer recorded the number of food-cup photobeam breaks during each CS and during the 30 -sec interval that immediately preceded it (the preperiod). Each trial's data were then converted into "elevation scores" by subtracting the data that were obtained during the preperiod from the data that were obtained during the CS. The data were analyzed with trend analyses (Keppel \& Zedeck, 1989), with a rejection criterion of $p<.05$.

\section{Results}

\section{Acquisition Phase}

An ANOVA comparing the acquisition of responding (elevation scores) in the groups as a function of sessions revealed an effect of session $[F(15,825)=8.45]$, but no interaction between group and session $[F(105,825)<1]$, suggesting little overall difference among groups in the rate at which responding developed over sessions. The elevation scores for each group averaged throughout the conditioning phase are therefore presented in the upper panel of Figure 1. The mean CS scores and scores from the preperiod are also shown. Notice that the $x$-axis corresponds to the ITI given the different groups (rather than trials or sessions) in order to highlight the quantitative effect of ITI. The figure suggests that - although responding in the shorter interval groups was somewhat more variable than expected given the results of previous experiments (Bouton \& Sunsay, 2003; Sunsay et al., 2004) - there was overall an increasing linear effect of ITI on the elevation scores. A trend analysis confirmed a significant linear effect $[F(1,56)=10.82]$. No other trends (e.g., quadratic, cubic, quartic) approached significance $\left[F_{\mathrm{S}}(1,56)<1\right]$. When we isolated groups with ITI values of $240-1,920$, the linear trend was also reliable $[F(1,35)=5.27]$. The pattern suggests that regardless of whether ITI values below $240 \mathrm{sec}$ are included or excluded, the effect of ITI averaged over sessions is best described by a straight line.

An analysis of the scores from the preperiod of all groups revealed a significant linear trend $[F(1,56)=5.85]$. However, there was also a significant quadratic trend $[F(1,56)=$ $8.00]$. Scores during the preperiod began to decrease from 480 -sec to 1,920 -sec ITIs, but not from 60 -sec to 240 -sec.

\section{Test Phase}

Responding to the CS was tested again after two sessions of context extinction that were designed to reduce group differences in responding in the preperiod. Although context extinction did not eliminate responding in the preperiod (consistent with the fact that it has a nonzero baseline level), it did eliminate group differences (see lower panel of Figure 1). Importantly, however, it did not substantially change the pattern of responding to the CS. Elevation scores of all groups on the test trials were again subjected to a trend analysis, which again revealed a significant linear trend $[F(1,56)=4.07]$ but no higher order trends $\left[F_{\mathrm{S}}(1,56)<1\right]$. An identical analysis on prescores revealed no significant trend of any kind $[F \mathrm{~s}(1,56) \leq 1.14]$. When we isolated the groups with ITIs of $240-1,920 \mathrm{sec}$, the linear trend was again reliable $[F(1,35)=7.56]$, with no higher order trends or differences in rates during the preperiod $\left(F_{\mathrm{S}}<1\right)$. The 

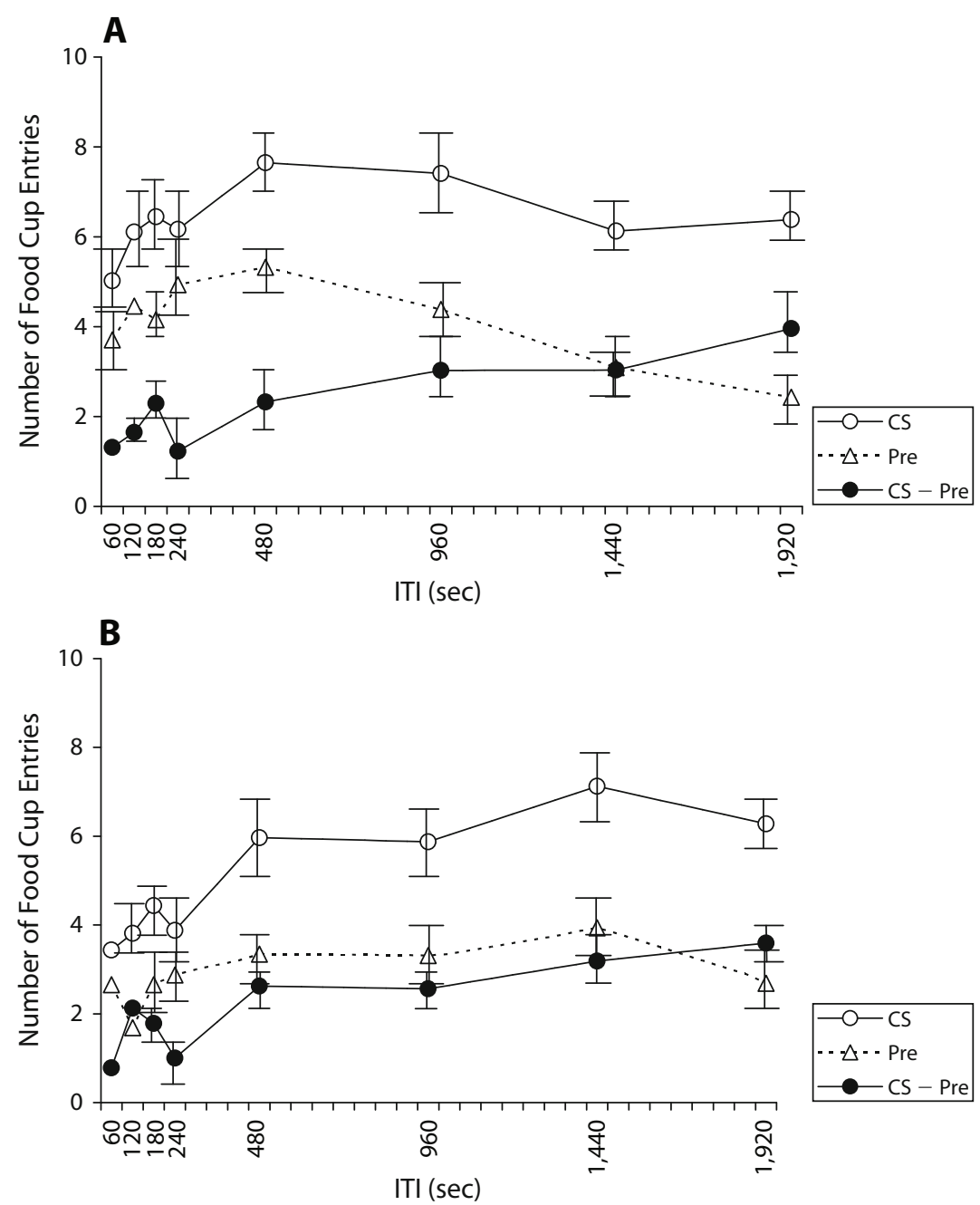

Figure 1. Panel A: Mean $( \pm S E M)$ number of food-cup entries over the ITI conditions during the acquisition sessions in Experiment 1. Panel B: Mean $( \pm S E M)$ number of food-cup entries over the ITI conditions during the test session in Experiment 1.

test results thus confirm that the present ITI values contribute in a linear fashion to responding to the CS.

\section{Discussion}

The results of this experiment suggest that trial-spacing effects do occur above the $240-\mathrm{sec}$ ITI. As emphasized in the introduction, previous results (Bouton \& Sunsay, 2003; Sunsay et al., 2004) suggest that the self-generated priming mechanism cannot account for a trial-spacing effect above $240 \mathrm{sec}$. Clearly, other mechanisms must therefore also operate to produce trial-spacing effects in this preparation. Overall, the results suggest that responding is a single linear function of trial spacing over the entire range of ITIs, as might be implied by quantitative accounts of trial spacing, which suggest that the animal's decision point should be a linear function of the ITI (i.e., as determined by I/T; Gallistel \& Gibbon, 2000; Gibbon \& Balsam, 1981). However, the linear relationship was also apparent during a test conducted after conditioning was finished, suggesting that the ITI might also influence the final level of responding. (Condi- tioned responding had reached an asymptote before the test: There was no session effect when we analyzed the groups over Sessions 9 through 16 [the last conditioning session prior to testing] $[F(7,385)<1]$.) Thus, ITI appears to influence the asymptotic level of conditioned responding over and above any effect it might have on the decision point.

The prescores during acquisition showed a significant curvilinear (quadratic) trend as a function of the ITI. Although there was no change in prescores from the 60 -sec to the $240-\mathrm{sec}$ or 480 -sec ITI conditions, preresponding tended to decrease as the ITI increased beyond $480 \mathrm{sec}$. This pattern is consistent with the idea that responding in the preperiod reflects the strength of the association between the context and the US. Lower responding should have occurred with the longer ITIs; more extinction of the context would be expected. Such a trend is anticipated by models implicating a context blocking account of the trialspacing effect (Pearce \& Hall, 1980; Rescorla \& Wagner, 1972; Wagner, 1978, 1981). The prescore differences did not complicate our interpretation of the effect of ITI on CS 
conditioning because the ITI effect was still evident (Figure 1 , lower panel) after they were eliminated by the context extinction sessions administered after conditioning.

\section{EXPERIMENT 2}

According to the context blocking mechanism, extinction of the context-US association during longer ITIs reduces the context's ability to block learning of the CS's association with the US. Barela (1999) tested this explanation in a fear-conditioning experiment by removing rats from the context during the ITI. Removal from the context should eliminate the possibility of context extinction and therefore increase context blocking, despite a long ITI. In a fear-conditioning paradigm, some rats were removed from the context during 900-sec ITIs, whereas other rats remained in the context. Barela found no evidence for context blocking of the CS when the CS was tested in a novel context; the removed and nonremoved groups had comparable freezing scores. We used a similar manipulation in Experiment 2 to test the role of context exposure in the present appetitive conditioning preparation. An important deviation from Barela's experiment was that there were two ITI conditions in this experiment designed to confirm the presence of a trial-spacing effect and assess the effect of context removal at both a short and a long ITI.

The design is illustrated in Figure 2. Two groups received conditioning with an ITI of $240 \mathrm{sec}$, and two other groups received an ITI of 1,440 sec. One group from each condition spent the ITI in the conditioning context, as is true in conventional conditioning procedures. However, one group from each condition was removed from the context and spent the bulk of the ITI in a control context. These groups received the same time between successive trials, but removal prevented context extinction from occurring. If context extinction contributes to the superior conditioning obtained with a long ITI, then removal from the context should reduce conditioning in the 1,440 -sec removed group.

\section{0 sec-Removed}

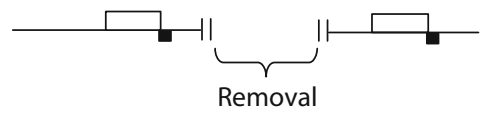

$240 \mathrm{sec}$

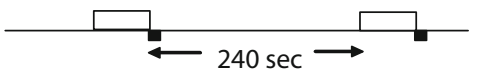

$1,440 \mathrm{sec}-$ Removed

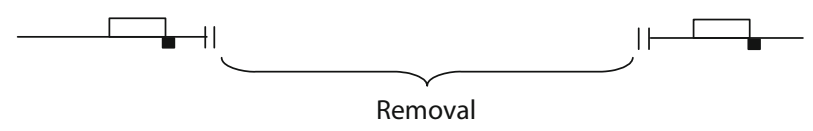

$1,440 \mathrm{sec}$

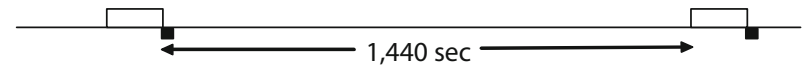

Figure 2. Design of Experiment 2.
After 14 sessions of acquisition, conditioned responding to the CS was tested in a different context to separate the associative strength of the CS from possible differences in the conditioned strength of the training context. Theoretical explanations of the trial-spacing effect that emphasize context extinction still predict higher responding to the CS in the groups that were not removed from the conditioning context during the ITIs.

\section{Method}

\section{Subjects and Apparatus}

The subjects were 32 female Wistar rats of the same age and stock as those in the preceding experiment. The apparatus, CS, and US were also the same. For half the rats, the chambers with horizontal stripes and anise odor served as the conditioning context, and the chambers with dots and coconut odor were the testing context. The other half received the reverse. The "removed" rats were put in $44.5 \times 23.8 \times$ $20 \mathrm{~cm}$ plastic shoebox cages with sawdust on the floor during the ITIs. These boxes were located in an adjacent room.

\section{Procedure}

Acquisition phase. The experiment began with two initial 20-min magazine training sessions in which the rats were put in the conditioning boxes to learn to retrieve pellets from the food cups. The magazine training took place both in the conditioning and the testing contexts (one session each). Each rat received 33-37 pellets in each initial session.

The rats were randomly assigned to four groups $(n=8)$ created by the factorial combination of two factors (ITI and context removal), with two levels each. Boxes were also counterbalanced. All rats then received two conditioning sessions a day (separated by $3 \mathrm{~h}$ ) for the next 7 days. There were four reinforced CS presentations in each session. For each group, the first CS was given 4 min into the session, and the session ended within a minute after the last conditioning trial. As illustrated in Figure 2, the rats received trials separated by a fixed 240 -sec or a fixed 1,440 -sec ITI. Half of the rats in each ITI condition were removed from the training context and placed in a shoebox cage $60 \mathrm{sec}$ after each US. They were then returned to the context $60 \mathrm{sec}$ before the next preperiod. The other half of the rats spent the ITI in the training context. To control for handling, these rats were removed from the context, placed briefly $(2-3 \mathrm{sec})$ in a shoebox cage, and then returned to the training context. This handling took place at either of the times the removal groups were handled (i.e., on being removed from the apparatus $60 \mathrm{sec}$ after each US and being returned to the apparatus $60 \mathrm{sec}$ before the next preperiod) in a double alternating schedule. Note that all groups were thus removed, placed in a shoebox, and then returned to the training context exactly once between each pair of conditioning trials. Note also that the controls were handled (on average) at the same times as the experimental groups. Interestingly, the performance of the control groups did not differ on the trials that followed the two handling times $[F(1,15)=2.36$, collapsed over all sessions of the phase $]$

Test phase. On the 8 th day, all rats were switched to the alternate context in which they had received only magazine training 8 days earlier. In a single test session, all groups then received eight nonreinforced tone presentations spaced by the geometric mean of the two ITIs (i.e., $588 \mathrm{sec}$ ). The geometric mean was used on the basis of studies suggesting that the geometric mean of two intervals is perceived as being equally distant from both intervals (Church \& Deluty, 1977).

\section{Results}

\section{Acquisition Phase}

The results of the acquisition phase are presented in Figure 3, which shows elevation scores over the seven twosession blocks of conditioning. Because of a procedural error during one ITI of the third session, half of the shortstay group was inappropriately removed from the context, 


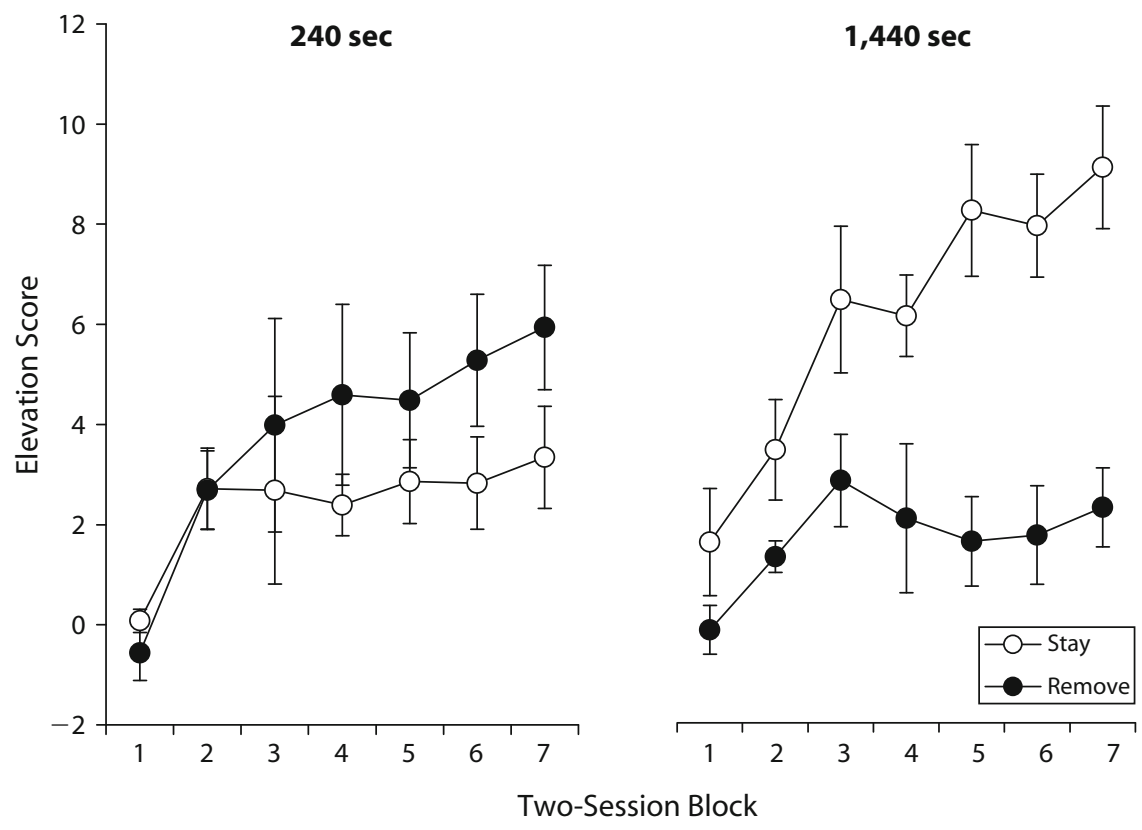

Figure 3. Mean $( \pm S E M)$ elevation scores (number of responses in the CS minus the number of responses in the preperiod) over two-session blocks during acquisition in Experiment 2.

and half of the short-remove group was inappropriately allowed to remain in the context. Data from the corresponding rats in this session were therefore excluded from the data analyses. (Figure 3 shows the mean of the unaffected animals.) For rats that stayed in the context, conditioning proceeded more rapidly with the 1,440 -sec ITI than with the 240-sec ITI. More importantly, however, removing the rat from the conditioning context reduced the facilitating effect of the 1,440-sec ITI. An ITI $\times$ removal $\times$ session block ANOVA revealed a significant session block effect $[F(6,168)=15.34]$. The removal main effect was also significant $[F(1,28)=4.55]$. The ITI main effect was not significant $[F(1,28)=1.51]$, but there was an interaction between ITI and removal $[F(1,28)=15.72]$. The pattern suggests that the removal effect was only evident in the long ITI condition. Planned comparisons confirmed this suggestion: There was no difference between the remove and stay groups with the 240 -sec ITI $[F(1,14)=1.89]$, but the corresponding groups differed significantly at the 1,440 -sec ITI $[F(1,14)=16.58]$. The session block $\times$ ITI $\times$ removal interaction was also significant $[F(6,168)=$ 3.78], whereas the ITI $\times$ removal interaction developed over sessions. No other effects were reliable $\left(F_{\mathrm{S}}<1\right)$.

An identical ANOVA on prescores revealed a nonsignificant session-block effect $[F(6,168)=1.57]$. There was a significant ITI effect $[F(1,28)=5.72]$; the short ITI groups had higher prescores. Neither the removal effect $[F(1,28)=1.32]$ nor the ITI $\times$ removal interaction $[F(1,28)<1]$ was reliable. A planned comparison failed to find a difference between the remove and the stay groups with the 1,440 -sec ITI $[F(1,28)=2.82]$. Other effects were also not reliable [the largest $F(12,336)=$ 1.52]. Inferences based on the removal $\times$ ITI interaction in the elevation scores were therefore not compromised by differences in the prescores. The mean prescores were $4.12,4.80,2.31$, and 3.26 for the 240-stay, 240-remove, 1,440-stay, and 1,440-remove groups, respectively.

A comparison of the 1,440-remove and the 240-remove groups is theoretically important. Since these groups spent an equal amount of time in the context, the context blocking explanation (for example) would predict no difference between them, even though they differed in the amount of time that elapsed between trials. Planned comparisons showed no significant difference between these groups $[F(1,14)=3.48]$. If anything, the nonsignificant trend was opposite to what one might have expected on the basis of the trial-spacing effect (the 240-remove group had numerically higher, rather than lower, elevation scores).

\section{Test Phase}

The rats then received nonreinforced trials in the test context in order to assess the associative strength of the CS separate from different possible associative strengths in the training context. The results of the test trials are presented in Figure 4 . An ITI $\times$ removal ANOVA revealed that the ITI and the removal main effects were not significant $[F \mathrm{~s}(1,28)<1]$. However, consistent with the results of the conditioning phase, the ITI $\times$ removal interaction was highly significant $[F(1,28)=6.32]$. Once again, the 1,440 -sec ITI group that was removed from the context during the ITI had lower elevation scores than did the group that stayed in the context. A planned comparison confirmed this $[F(1,28)=4.56]$. There was no difference between the two groups that had been given the shorter ITI $[F(1,28)=2.03]$.

An identical ANOVA on scores from the preperiod revealed no reliable effects [largest $F(1,28)=2.02$ ]. The mean number of responses in the preperiod were 1.98 , 


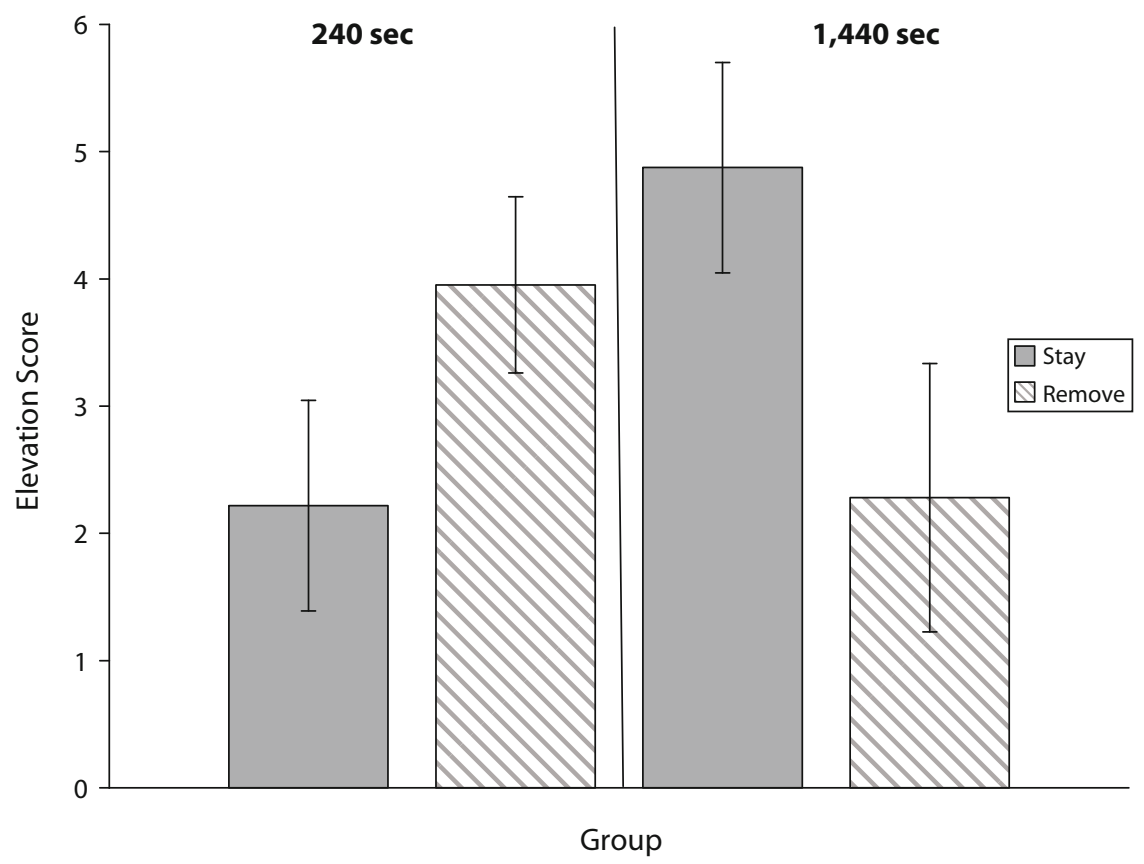

Figure 4. Mean ( $(S E M)$ elevation scores (number of responses in the CS minus the number of responses in the preperiod) during the test phase of Experiment 2.

$1.39,1.23$, and 0.59 for groups $240-$ stay, $240-$ remove, 1,440-stay, and 1,440-remove, respectively.

\section{Discussion}

Removing rats from the context during the ITI eliminated the benefit of conditioning with the long ITI. This result suggests that exposure to the context during the ITI plays a crucial role in the trial-spacing effect with long ITIs in this preparation. The effect of removing the rat from the context was limited to the long $(1,440-\mathrm{sec})$ ITI. Presumably, exposure to the context at the shorter 240 -sec interval was not sufficient to extinguish the context, and the results thus suggest that the context blocking mechanism would play little role in trial-spacing effects of ITIs under this value. This possibility might leave the self-generated priming mechanisms (which we previously identified) as the cause of trial-spacing effects below $240 \mathrm{sec}$ (Bouton \& Sunsay, 2003; Sunsay et al., 2004).

The present effect of context removal is not consistent with the findings of Barela (1999) in fear conditioning. Barela found that removing rats from the conditioning context prevented extinction of the context, but that it did not worsen conditioning with the CS. It is possible that the ITI values used in Barela's study were not conducive to detecting context blocking in the fear conditioning preparation. Alternatively, as Barela himself argued, it is possible that the results with fear conditioning depended on a specific neural interaction (a short-term inhibition of the amygdala by the dorsolateral periacqueductal gray after exposure to footshock) that would not play a role outside the domain of fear conditioning.

The present findings are consistent, however, with other results in the literature. One effect of the removal manipulation was to create a local exposure to the training context that preceded and followed each conditioning trial by $60 \mathrm{sec}$. Previous experiments have studied the effects of a similar "local context" created by presenting a CS in a similar temporal relationship with the target CS-US pairing (e.g., Barnet, Grahame, \& Miller, 1993). The results of those studies suggest that the local context competes with conditioning of the CS; when the local context is paired with the US prior to conditioning, it interferes with conditioning to the target CS. Most important, extinction exposure to the local context then increases responding to the target (Barnet et al., 1993). The effect of exposure to the context between trials in the present control groups, which was likewise to increase responding to the CS, is analogous to that finding.

The response-enhancing effect of context exposure during the ITI is consistent with several accounts-such as the context blocking explanation (Pearce \& Hall, 1980; Rescorla \& Wagner, 1972; Wagner, 1978, 1981; Wagner \& Brandon, 1989, 2001) or the comparator hypothesis - that make explicit reference to competition between CS-US and context-US associations. According to context blocking, extinction exposure to the context during the long ITI would weaken the context's ability to block conditioning of the CS-US association. According to the comparator hypothesis, extinction exposure to the context would reduce its ability to suppress the CS-US association's manifestation in performance (see Barnet et al., 1993). A timeaccumulation model (Gallistel \& Gibbon, 2000) would need to assume that the internal clock that allows estimation of reinforcement rate in the background (I) stops when the rat is removed from the physical conditioning context and then starts again when the animal is returned. Of course, that assumption would be required whenever a trial-spacing effect is observed over multiple conditioning 
sessions separated, for example, by $24 \mathrm{~h}$ (see, e.g., Bouton $\&$ Sunsay, 2003; Sunsay et al., 2004). The results of this experiment indicate that the trial-spacing effect with long ITIs in this conditioning preparation depends on the rat being in the conditioning context during the long ISI.

\section{EXPERIMENT 3}

The third experiment was designed to test for a trialspacing effect with a novel within-subjects design. The main virtue of the design was that it equated the strength of any context-US association that was present when the massedtrial and spaced-trial CSs were paired with the US. For example, consider CSs A and B presented and reinforced in the same context in a sequence such as ABBA. If each successive trial is separated by the same ITI, then the associative strength of the context should be the same whenever A or B is reinforced. The amount of context blocking (see, e.g., Rescorla \& Wagner, 1972), the strength of the comparator term (Miller \& Matzel, 1988; Miller \& Schachtman, 1985), or the estimate of reinforcement rate in the background (Gallistel \& Gibbon, 2000) should thus be equivalent for CSs A and $\mathrm{B}$. Therefore, if A and B are equally salient, then they should be learned equally well, even though trials with $B$ are relatively massed, and trials with A are relatively spaced.

On the other hand, in an ABBA sequence, successive presentations of CS A are nonetheless separated by more time (and more context exposure) than are successive presentations of CS B. As was noted in the introduction, SOP (see, e.g., Wagner, 1981) uniquely predicts that context$\mathrm{CS}$ associations might also contribute to trial-spacing effects. Although the ABBA arrangement controls for the strength of context-US associations that are present when either CS is reinforced, the longer interval between the two CS A presentations would allow more weakening of the context-A association than of the context-B association. This would reduce retrieval-generated priming of CS A and allow better conditioning of A than of B. In Experiment 3, therefore, we asked whether a trial-spacing effect still occurred under conditions that would appear to isolate a role for retrieval-generated priming of the CS.

\section{Method}

\section{Subjects and Apparatus}

The experiment was run in two replications (repetitions of the experiment), each involving 16 female Wistar rats of the same age and stock as those in the preceding experiments. The apparatus and housing conditions were also the same. The tone used in Experiment 1 also served as one of the CSs. As before, the tone was delivered through a speaker positioned above the ceiling of the chamber. The other CS was a 70-dBA clicker (which clicked every half second) that was delivered through a speaker mounted on the rear side wall of each chamber. These two CSs were counterbalanced as CSs A and B. Turning the houselights off was used as a dummy CS, Stimulus C. All trials were reinforced with the two-pellet US that used in the preceding experiments.

\section{Procedure}

Acquisition phase. The experiment began with an initial 20-min magazine training session in which the rats were put in the boxes to learn to retrieve pellets from the food cups. As in previous experiments, the magazine training took place in two contexts. Each rat received 31-32 pellets in each initial session.

There were then sixteen 90-min conditioning sessions, each using one of the eight different trial schedules presented in Table 1. The schedule for each session was chosen randomly without replacement from this set, and the resulting order of eight schedules (i.e., 1, 3, $8,5,2,4,7$, and 6 ) was repeated twice. There was a total 8 days of conditioning, with two sessions separated by $3 \mathrm{~h}$ in each. In each session there were three A trials, three B trials, and nine C trials, all reinforced. Each trial was separated from the previous one by an average 330 -sec ITI (varying $25 \%$ around the mean). The dummy lights-off CS (C) served two purposes. First, it filled the ITI between some of the spaced trials (i.e., ACCCA). Second, it served to prevent the confounding of stimulus spacing (i.e., spaced or massed) with the trial position in the session. That is, over sessions, A and B occupied the various trial positions the same number of times. Stimuli $\mathrm{A}$ and $\mathrm{B}$ were clicker and tone for half of the subjects (Group CTTC) and tone and clicker for the other half (Group TCCT). The acquisition data were analyzed over two-session blocks (or days).

Test phase. In the second replication $(n=16)$, a test phase was introduced. (Events external to the experiment precluded such a test for the first replication.) During the test phase, A and B were each tested without the US in the alternate context over a series of eight 30-min sessions. Each test session consisted of five trials separated by ITIs of $330 \mathrm{sec}$. The first trial was always the lights-off (dummy) CS followed by the US. For half of the rats, the next four trials in the first four sessions were ABBA; for the other half, they were BAAB. The rats were given the opposite order for the second four sessions. The sessions were separated by a 1-h interval.

\section{Results}

\section{Acquisition Phase}

The results are presented in Figure 5, which shows the mean elevation scores over two-session blocks for the massed and spaced CS. The results of the two replications were highly consistent. A spacing $\times$ stimulus assignment $\times$ block $\times$ replication ANOVA revealed no main

Table 1

Trial Schedules Used in Experiment 3

\begin{tabular}{|c|c|c|c|c|c|c|c|c|c|c|c|c|c|c|c|}
\hline & $\mathrm{P} 1$ & P2 & P3 & P4 & P5 & P6 & P7 & P8 & P9 & $\mathrm{P} 10$ & P11 & P12 & P13 & P14 & P15 \\
\hline 1 & $\mathrm{C}$ & $\mathbf{A}$ & B & B & B & $\mathbf{A}$ & $\mathrm{C}$ & $\mathrm{C}$ & $\mathrm{C}$ & $\mathbf{A}$ & $\mathrm{C}$ & $\mathrm{C}$ & $\mathrm{C}$ & $\mathrm{C}$ & $\mathrm{C}$ \\
\hline 2 & $\mathrm{C}$ & B & B & B & $\mathbf{A}$ & $\mathrm{C}$ & $\mathrm{C}$ & $\mathrm{C}$ & $\mathbf{A}$ & $\mathrm{C}$ & $\mathrm{C}$ & $\mathrm{C}$ & $\mathbf{A}$ & $\mathrm{C}$ & $\mathrm{C}$ \\
\hline 3 & $\mathrm{C}$ & $\mathrm{C}$ & $\mathbf{A}$ & $\mathrm{C}$ & $\mathrm{C}$ & $\mathrm{C}$ & $\mathbf{A}$ & B & B & B & $\mathbf{A}$ & $\mathrm{C}$ & $\mathrm{C}$ & $\mathrm{C}$ & $\mathrm{C}$ \\
\hline 4 & $\mathrm{C}$ & $\mathrm{C}$ & $\mathbf{A}$ & $\mathrm{C}$ & $\mathrm{C}$ & $\mathrm{C}$ & $\mathbf{A}$ & $\mathrm{C}$ & $\mathrm{C}$ & $\mathrm{C}$ & $\mathbf{A}$ & B & B & B & $\mathrm{C}$ \\
\hline 5 & $\mathrm{C}$ & $\mathrm{C}$ & $\mathrm{C}$ & $\mathbf{A}$ & B & B & B & $\mathbf{A}$ & $\mathrm{C}$ & $\mathrm{C}$ & $\mathrm{C}$ & $\mathbf{A}$ & $\mathrm{C}$ & $\mathrm{C}$ & $\mathrm{C}$ \\
\hline 6 & $\mathrm{C}$ & $\mathrm{C}$ & $\mathrm{C}$ & $\mathbf{A}$ & $\mathrm{C}$ & $\mathrm{C}$ & $\mathrm{C}$ & $\mathbf{A}$ & B & B & B & $\mathbf{A}$ & $\mathrm{C}$ & $\mathrm{C}$ & $\mathrm{C}$ \\
\hline 7 & $\mathrm{C}$ & $\mathrm{C}$ & $\mathrm{C}$ & $\mathrm{C}$ & $\mathbf{A}$ & B & B & B & $\mathbf{A}$ & $\mathrm{C}$ & $\mathrm{C}$ & $\mathrm{C}$ & $\mathbf{A}$ & $\mathrm{C}$ & $\mathrm{C}$ \\
\hline 8 & $\mathrm{C}$ & $\mathrm{C}$ & $\mathrm{C}$ & $\mathrm{C}$ & $\mathrm{C}$ & $\mathbf{A}$ & $\mathrm{C}$ & $\mathrm{C}$ & $\mathrm{C}$ & A & B & B & B & $\mathbf{A}$ & $\mathrm{C}$ \\
\hline
\end{tabular}

Note-P, trial position; A, spaced CS; B, massed CS; C, dummy CS 


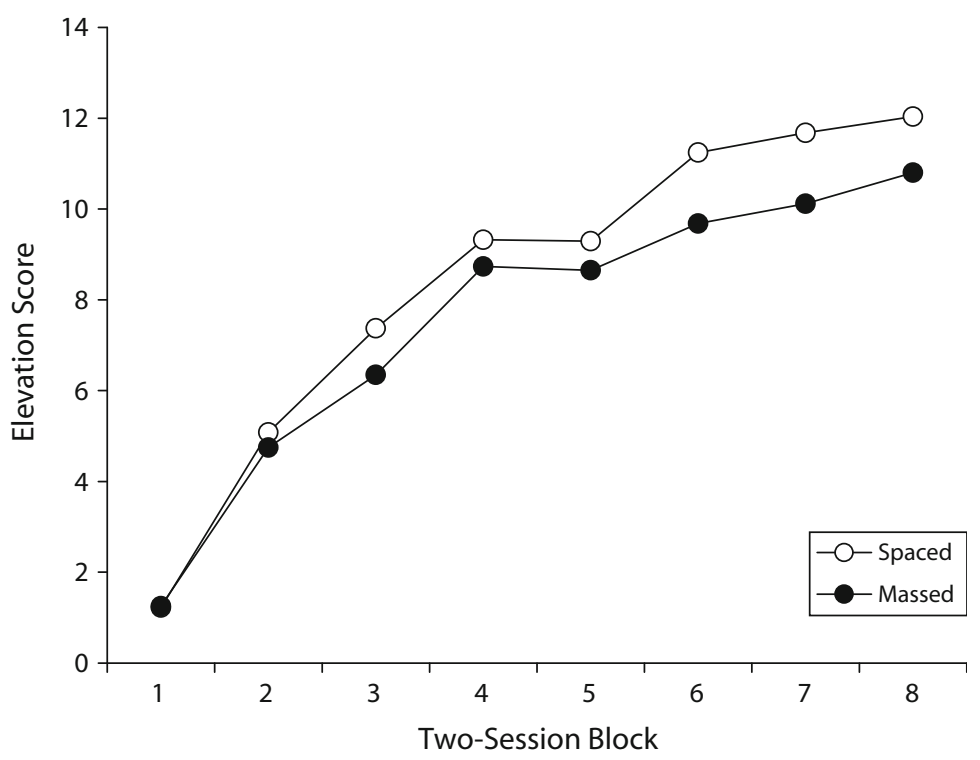

Figure 5. Mean elevation scores (CS minus pre) during two-session blocks of acquisition in Experiment 3.

effect or interaction involving the replication factor, the largest $[F(1,28)=2.13]$. However, there was a significant main effect of block $[F(7,196)=40.57]$ and, most importantly, there was a highly significant main effect of trial spacing $[F(1,28)=12.02]$. Thus, there was more responding to the CS that underwent spaced presentations than to the CS that underwent massed presentations. The effect of spacing interacted with stimulus assignment $[F(1,28)=$ 17.66]; the effect was more pronounced when the clicker was the spaced stimulus. On the final two sessions, Group CTTC had mean elevation scores of 14.42 and 11.31 to the spaced clicker and massed tone, whereas Group TCCT had mean elevation scores of 12.50 and 11.15 to the spaced tone and massed clicker. The clicker was more salient than the tone $[F(1,30)=13.19]$. The pattern and main effect of spacing indicate that the longer ITI supported more responding than did the shorter ITI during conditioning.

A corresponding ANOVA on the prescores did not reveal significant effects of either block $[F(7,196)=1.40]$ or spacing $[F(1,28)=3.17]$. Nor was there an effect of stimulus assignment or replication, or an interaction between these factors $\left[F_{\mathrm{s}}(1,28) \leq 1.68\right]$. There was a significant stimulus assignment by block effect $[F(7,196)=$ $2.16]$. There was also a replication $\times$ block interaction $[F(7,196)=4.69]$. The mean prescores collapsed over blocks and group were 3.69 and 4.04 for the spaced and massed trials, respectively.

\section{Test Phase}

The rats in the second replication then received the CSs in the alternate context with both ABBA and BAAB orders. This meant that both the massed and spaced CSs were tested in massed and spaced arrangements. Figure 6 summarizes the results of testing, which strongly suggest the influence of trial spacing during conditioning but not during testing. An acquisition spacing $\times$ test spacing $\times$ stimulus assignment $\times$ test order ANOVA revealed that the critical acquisition spacing main effect was once again significant $[F(1,12)=7.53]$. Thus, the CS that had received spaced training still evoked more responding than the CS that had received massed training. In contrast, the test spacing effect $[F(1,12)<1]$ and the acquisition spacing $\times$ test spacing interaction $[F(1,12)=2.35]$ were not reliable. There was a marginal effect of stimulus assignment $[F(1,12)=3.85, p=.073]$, with slightly more responding in subjects for which the clicker had received spaced conditioning. Stimulus assignment similarly interacted with acquisition spacing $[F(1,12)=5.42]$, and the stimulus assignment $\times$ acquisition spacing $\times$ test spacing was also reliable $[F(1,12)=6.86]$. The test order $\times$ acquisition spacing $\times$ stimulus assignment effect approached significance $[F(1,12)=3.89, p=.072]$. No other effects approached significance [the largest $F(1,12)=2.26$ ]. The pattern of results suggested that the effect of spacing trials that was evident in acquisition was still present during testing, regardless of the spacing of the test trials.

A similar analysis performed on the prescores did not reveal an effect of stimulus assignment, acquisition spacing, or test order $(F \mathbf{S}<1)$. There was an acquisition spacing $\times$ stimulus assignment $\times$ test order effect $[F(1,12)=$ 7.78]. The other effects were not reliable [the largest $F(1,12)=3.77]$. The mean prescores for the group given spaced clickers (Group CTTC) were 2.00 and 1.73, respectively, for clicker and tone; the corresponding scores were 2.08 and 2.02 for Group TCCT.

\section{Discussion}

The results revealed a trial-spacing effect despite the use of a within-subjects design that controlled for the strength of context-US associations in the presence of the massed and the spaced trials. Tests conducted in the alternate context produced entirely consistent results; a difference in 


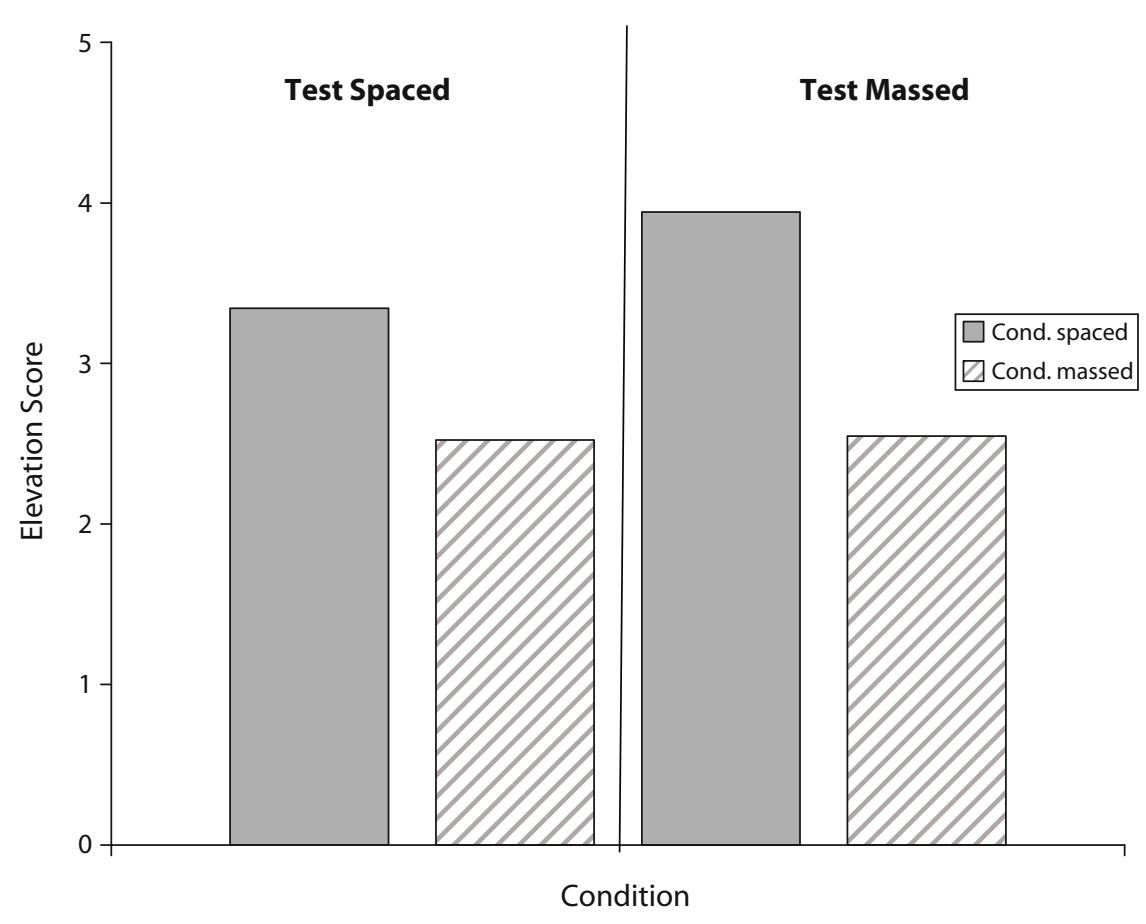

Figure 6. Mean elevation scores (CS minus pre) during the test phase in Experiment 3.

responding was still observed in the different context, regardless of whether the CSs were now spaced or massed.

The trial-spacing effect obtained in this experiment presents difficulties for most explanations of the trial-spacing effect. Because the associative strength or the reinforcement rate of the context was controlled over spaced and massed trials in the present within-subjects design, the context blocking (Pearce \& Hall, 1980; Rescorla \& Wagner, 1972; Wagner, 1978, 1981; Wagner \& Brandon, 1989, 2001), comparator hypothesis (Miller \& Matzel, 1988; Miller \& Schachtman, 1985), and time-accumulation models (Gallistel \& Gibbon, 2000; Gibbon \& Balsam, 1981) predicted no difference in conditioning with the present massed and spaced CSs. Nonetheless, according to the retrievalgenerated priming mechanism in SOP (Wagner, 1981; Wagner \& Brandon, 1989, 2001), the context-B association should be stronger than the context-A association, which would make it more difficult to activate CS B to the A1 state during conditioning. Thus, the results of Experiment 3 may uniquely point to the role of the extinction of context-CS associations in the explanation of the trial-spacing effect.

\section{GENERAL DISCUSSION}

The present experiments extended previous research in this laboratory that investigated trial-spacing effects in appetitive conditioning (Bouton \& Sunsay, 2003; Sunsay et al., 2004). As reviewed in the introduction, that research did not support the account provided by time accumulation models (Bouton \& Sunsay, 2003; Sunsay et al., 2004) and instead suggested the importance of self-generated priming mechanisms like those anticipated by SOP (see, e.g., Wagner, 1981). However, the research also suggested that self-generated priming has an upper bound of less than $240 \mathrm{sec}$ in the present conditioning preparation (Bouton \& Sunsay, 2003; Sunsay et al., 2004). Therefore, any trial-spacing effect that occurs above a 240 -sec ITI would need to be explained by other mechanisms.

The results of each of the present experiments suggest that a trial-spacing effect does occur above the 240 -sec value in this preparation. The results of Experiment 1 suggested that a trial-spacing effect can occur in the range of 480- to 1,920 -sec intervals and further indicated that the effect of ITI over the entire range of $60-1,920 \mathrm{sec}$ was best described as a linear function. This was true despite the fact that the effects of different ITIs above and below $240 \mathrm{sec}$ appear to be controlled by different mechanisms (see, e.g., Sunsay et al., 2004; present Experiment 2). The overall pattern suggests that it may be misleading to assume that the discovery of a simple quantitative relationship between conditioning and an ITI parameter (e.g., I or I/T) necessarily implies the operation of a single psychological mechanism over all ranges of ITI (cf. Gallistel \& Gibbon, 2000). Since the ITI effect was evident in a test phase that was conducted after responding had reached its asymptote, ITI appeared to affect the level of responding over and above any effect it might have had on the point at which the animal decided to respond (cf. Gallistel \& Gibbon, 2000).

The results of Experiment 2 indicated that exposure to the context during the ITI plays a crucial role in controlling the trial-spacing effect with long ITIs. Specifically, removing rats from the context during a 1,440-sec ITI abolished the advantage that this ITI conferred on conditioning relative to a 240 -sec ITI. The effect of context removal was observed on responding during the course of acquisition and during final tests conducted with a different ITI in a 
different context. The clear effect of context removal contrasts with Barela's (1999) results in fear conditioning (the conditioned freezing preparation), and one must note that Experiment 2 differed from Barela's experiment in many ways. However, as Barela argued, the results with fear conditioning might depend on the interaction between specific brain mechanisms that may be uniquely implicated in fear conditioning (the dorsolateral periaqueductal gray and amygdala). The present results clearly implicate the importance of context exposure during long ITIs in the present appetitive conditioning preparation.

In principle, the importance of context exposure during the ITI is consistent with several accounts of the trialspacing effect. According to the context blocking account (see, e.g., Rescorla \& Wagner, 1972; Wagner, 1981), context exposure during long ITIs would extinguish contextual conditioning and thus reduce the context's ability to block learning about the CS. According to the comparator hypothesis (e.g., Miller \& Schachtman, 1985), context exposure would also extinguish contextual conditioning; however, in this case, it would reduce the effect of the comparator stimulus on performance on the basis of a strong CS-US association. The present experiments do not distinguish between these two types of accounts. However, it is worth noting that Sunsay (2004) failed to confirm the comparator theory's prediction of an effect of extensive context exposure on performance to the CS when it was administered after conditioning when a short (240-sec) ITI had occurred (see also Holland, 1999). Finally, as we noted earlier, a time-accumulation model (Gallistel \& Gibbon, 2000) would predict the role of context exposure only if it assumed that the internal clock that estimates reinforcement rate in the absence of the CS runs only in the context in which conditioning occurs. Such an assumption is also required in tests of the effects of the $\mathrm{I} / \mathrm{T}$ ratio, because the calculation of $\mathrm{I} / \mathrm{T}$ usually excludes the intersession interval. However, given the fact that other work in this laboratory (Bouton \& Sunsay, 2003; Sunsay et al., 2004) has questioned a time-accumulation account of conditioning, our overall results with trial-spacing manipulations tend to favor trial-based models in which time is included as a factor (e.g., Wagner, 1981).

Nonetheless, Experiment 3 produced evidence of a trialspacing effect that cannot be attributed to differences in the strength of the context-US association. In that experiment, we obtained a trial-spacing effect using a withinsubjects design that explicitly controlled for the strength of the context-US association and the rate of reinforcement in the background for both massed and spaced CSs. The results were thus not anticipated by the context blocking account (Pearce \& Hall, 1980; Rescorla \& Wagner, 1972; Wagner, 1978, 1981; Wagner \& Brandon, 1989, 2001), the comparator hypothesis (Miller \& Matzel, 1988; Miller \& Schachtman, 1985; but see below), or time-accumulation models (Gallistel \& Gibbon, 2000; Gibbon \& Balsam, 1981). It is also worth emphasizing that the time between the "massed" trials in that experiment $(330 \mathrm{sec})$ was longer than the 240-sec window in which self-generated priming effects appear to operate in this preparation (Bouton $\&$ Sunsay, 2003; Sunsay et al., 2004). At the present point in time, the results of Experiment 3 are mainly consistent with the retrieval-generated priming of the CS mechanism that is suggested in SOP (see, e.g., Wagner, 1981). However, two qualifications are in order. First, it is possible that the comparator hypothesis can also accommodate Experiment 3's results, because it assumes that a CScontext association must be intact if the CS is to retrieve its comparator (contextual) stimulus - and thus the crucial comparator-US association - during testing. However, in order to explain the results of Experiment 3, the hypothesis would need to explain why being in the physical presence of the comparator stimulus during the test is not itself sufficient to retrieve the comparator-US association. It would also need to assume a "backward extinction" process in which exposure to the second term in the association (the context) weakens the CS-context association (ordinary extinction involves exposure to the first term). As a second qualification concerning the results of Experiment 3, it is worth noting that the results do not necessarily exclude a role for context-US associations in other examples of the trial-spacing effect. The context exposure manipulation studied in Experiment 2 would have theoretically affected both context-CS and context-US associations.

In summary, the present results, combined with previous research in this laboratory (Bouton \& Sunsay, 2003; Sunsay et al., 2004), suggest that trial-spacing effects in this conditioning preparation are consistent with mechanisms envisioned by SOP theory (see, e.g., Wagner, 1981; Wagner \& Brandon, 1989, 2001). With ITIs less than $240 \mathrm{sec}$, a recent presentation of CS or US may harm performance or learning because of self-generated priming of the stimulus in short-term memory (A2) (Bouton \& Sunsay, 2003; Sunsay et al., 2004). With ITIs over $240 \mathrm{sec}$, in which evidence suggests the self-generated priming mechanism cannot operate, the findings reported in the present article suggest that increased context exposure plays a crucial role. Although the results of Experiment 3 propose that context exposure may reduce retrieval-generated priming of the CS, it seems possible that, under ordinary conditions, it might also reduce retrieval-generated priming of the US, given evidence that context-US associations as indexed by responding in the preperiod decreases over the ITI range investigated in the present article (e.g., Experiment 1).

\section{AUTHOR NOTE}

This research was supported by Grant RO1 MH64847 from the National Institute of Mental Health to M.E.B. The experiments were part of a dissertation that C.S. submitted in partial fulfillment of the requirements for the Ph.D. degree at the University of Vermont. He thanks Kentaro Murakami, David J. Bucci, William A. Falls, and John T. Green for their helpful questions, comments, and suggestions, and Michael George for his technical assistance. Correspondence concerning this article should be addressed to C. Sunsay, 1101 E. 10th St., Department of Psychology, Indiana University, Bloomington, IN 47405 (e-mail: csunsay@indiana.edu).

Note-This article was accepted by the previous editorial team, when Shepard Siegel was Editor.

\section{REFERENCES}

BARELA, P. B. (1999). Theoretical mechanisms underlying the trialspacing effect in Pavlovian conditioning. Journal of Experimental Psychology: Animal Behavior Processes, 25, 177-193. 
Barnet, R. C., Grahame, N. J., \& Miller, R. R. (1993). Local time horizons in Pavlovian learning. Journal of Experimental Psychology: Animal Behavior Processes, 19, 215-230.

Bouton, M. E., \& Sunsay, C. (2003). Importance of trials versus accumulating time across trials in partially reinforced appetitive conditioning. Journal of Experimental Psychology: Animal Behavior Processes, 29, 62-77.

Church, R. M., \& Deluty, M. Z. (1977). Bisection of temporal intervals. Journal of Experimental Psychology: Animal Behavior Processes, 3, 216-228.

Domuan, M. (1980). Effects of intertrial interval on taste aversion learning in rats. Physiology \& Behavior, 25, 117-125.

DurLach, P. J. (1984). The effect of intertrial conditioned stimuli in autoshaping. Quarterly Journal of Experimental Psychology, 36B, 353-369.

DuRlach, P. J. (1989). Learning and performance in Pavlovian conditioning: Are failures of contiguity failures of learning or performance? In S. B. Klein \& R. R. Mowrer (Eds.), Contemporary learning theories: Pavlovian conditioning and the status of traditional learning theory (pp. 19-59). Hillsdale, NJ: Erlbaum.

EWING, M. F., LAREW, M. B., \& Wagner, A. R. (1985). Distributionof-trials effects in Pavlovian conditioning: An apparent involvement of inhibitory backward conditioning with short intertrial intervals. Journal of Experimental Psychology: Animal Behavior Processes, 11, 537-547.

Gallistel, G. R., \& Gibbon, J. (2000). Time, rate, and conditioning. Psychological Review, 107, 289-344.

Gibbon, J., Baldock, M. D., Locurto, C. M., Gold, L., \& Terrace, H. S. (1977). Trial and intertrial durations in autoshaping. Journal of Experimental Psychology: Animal Behavior Processes, 3, 264-284.

Gibbon, J., \& Balsam, P. (1981). Spreading associations in time. In C. M. Locurto, H. S. Terrace, \& J. Gibbon (Eds.), Autoshaping and conditioning theory (pp. 219-253). New York: Academic Press.

GlenberG, A. M. (1976). Monotonic and nonmonotonic lag effects in paired-associate and recognition memory paradigms. Journal of Verbal Learning \& Verbal Behavior, 15, 1-16.

GlenberG, A. M. (1979). Component-levels theory of the effects of spacing repetitions on recall and recognition. Memory \& Cognition, 7, 95-112.

GraU, J. W., \& Rescorla, R. A. (1984). The role of context in autoshaping. Journal of Experimental Psychology: Animal Behavior Processes, 10, 324-332.

Holland, P. C. (1999). Intertrial interval effects in Pavlovian serial feature positive discriminations. Animal Learning \& Behavior, 27, 127-139.

HoLland, P. C. (2000). Trial and intertrial durations in appetitive conditioning in rats. Animal Learning \& Behavior, 28, 121-135.

Jenkins, H. M., BARNES, R. A., \& BARRERA, F. J. (1981). Why autoshaping depends on trial spacing. In C. M. Locurto, H. S. Terrace, \& J. Gibbon (Eds.), Autoshaping and conditioning theory (pp. 255-284). New York: Academic Press.

KAPLAN, P. S. (1984). Importance of relative temporal parameters in trace autoshaping: From excitation to inhibition. Journal of Experimental Psychology: Animal Behavior Processes, 10, 113-126.

KePpel, G., \& ZeDECK, S. (1989). Data analysis for research design: Analysis of variance and multiple regression/correlation approaches. New York: Freeman.

KirKPATRICK, K., \& ChURCH, R. M. (2000). Independent effects of stimulus and cycle duration in conditioning: The role of timing processes. Animal Learning \& Behavior, 28, 373-388.

LatTAL, M. K. (1999). Trial and intertrial durations in Pavlovian conditioning: Issues of learning and performance. Journal of Experimental Psychology: Animal Behavior Processes, 25, 433-450.

Melton, A. W. (1970). The situation with respect to the spacing of repetitions and memory. Journal of Verbal Learning \& Verbal Behavior, 9, 596-606.

Miller, R. R., \& Matzel, L. D. (1988). The comparator hypothesis: A response rule for the expression of associations. In G. H. Bower (Ed.), The psychology of learning and motivation (Vol. 22, pp. 51-92). San Diego: Academic Press.

Miller, R. R., \& Schachtman, T. R. (1985). The several roles of context at the time of retrieval. In P. D. Balsam \& A. Tomie (Eds.), Context and learning (pp. 167-194). Hillsdale, NJ: Erlbaum.
Papini, M. R., \& Brewer, M. (1994). Response competition and the trial-spacing effects in autoshaping with rats. Learning \& Motivation, 25, 201-215.

Pearce, J. M., \& Hall, G. (1980). A model for Pavlovian learning: Variations in the effectiveness of conditioned but not of unconditioned stimuli. Psychological Review, 87, 532-552.

Phelan, L. L., Rodd, Z. A., Byers, D., \& Rosellini, R. A. (1998). Odor passive avoidance learning in individual Drosophila melanogaster: Parametric investigations of unconditioned stimulus intensity and inter-trial-interval. Learning \& Motivation, 29, 83-101.

Randich, A., \& Ross, R. T. (1985). Mechanisms of blocking by contextual stimuli. Learning \& Motivation, 15, 106-117.

Rescorla, R. A., \& Durlach, P. J. (1987). The role of context in intertrial interval effects in autoshaping. Quarterly Journal of Experimental Psychology, 39B, 35-40.

Rescorla, R. A., Durlach, P. J., \& Grau, J. W. (1985). Contextual learning in Pavlovian conditioning. In P. D. Balsam \& A. Tomie (Eds.), Context and learning (pp. 23-56). Hillsdale, NJ: Erlbaum.

Rescorla, R. A., \& Wagner, A. R. (1972). A theory of Pavlovian conditioning: Variations in the effectiveness of reinforcement and nonreinforcement. In A. H. Black \& W. F. Prokasy (Eds.), Classical conditioning II: Current research and theory (pp. 64-99). New York: Appleton-Century-Crofts.

Rogers, R. F., Talk, A. C., \& Matzel, L. D. (1994). Trial-spacing effects in Hermissenda suggest contributions of associative and nonassociative cellular mechanisms. Behavioral Neuroscience, 108, 1030-1042.

Salafia, W. R., Mis, F. W., Terry, W. S., Bartosiak, R. S., \& Daston, A. P. (1973). Conditioning of the nictitating membrane response of the rabbit (Oryctolagus cuniculus) as a function of length and degree of variation of intertrial interval. Animal Learning \& Behavior, 1, 109-115.

Spence, K. W., \& Norris, E. B. (1950). Eyelid conditioning as a function of the intertrial interval. Journal of Experimental Psychology, 40, 716-720.

SunsAY, C. (2004). A theoretical investigation of the trial-spacing effect in appetitive Pavlovian conditioning. Unpublished doctoral dissertation, University of Vermont, Burlington.

Sunsay, C., Stetson, L., \& Bouton, M. E. (2004). Memory priming and trial-spacing effects in Pavlovian learning. Learning \& Behavior, 32, 220-229.

Terrace, H. S., Gibbon, J., Farrell, L., \& Baldock, M. D. (1975). Temporal factors influencing the acquisition and maintenance of an autoshaped keypeck. Animal Learning \& Behavior, 3, 53-62.

WAGNER, A. R. (1978). Expectancies and the priming of STM. In S. H. Hulse, H. Fowler, \& W. K. Honig (Eds.), Cognitive processes in animal behavior. Hillsdale, NJ: Erlbaum.

WAGNER, A. R. (1981). SOP: A model of automatic memory processing in animal behavior. In N. E. Spear \& R. R. Miller (Eds.), Information processing in animals: Memory mechanisms (pp. 5-47). Hillsdale, NJ: Erlbaum.

WAGNER, A. R., \& BRANDON, S. E. (1989). Evolution of a structured connectionist model of Pavlovian conditioning (SOP). In S. B. Klein \& R. R. Mowrer (Eds.), Contemporary learning theories: Pavlovian conditioning and the status of traditional learning theory (pp. 149-189). Hillsdale, NJ: Erlbaum.

Wagner, A. R., \& BRANDON, S. E. (2001). A componential theory of Pavlovian conditioning. In S. B. Klein \& R. R. Mowrer (Eds.), Handbook of contemporary learning theories (pp. 23-64). Mahwah, NJ: Erlbaum.

Yeo, A. (1976). The acquisition of conditioned emotional response as a function of intertrial interval. Quarterly Journal of Experimental Psychology, 28, 449-458.

Yin, H., Barnet, R. C., \& Miller, R. R. (1994). Trial spacing and trial distribution effects in Pavlovian conditioning: Contributions of a comparator mechanism. Journal of Experimental Psychology: Animal Behavior Processes, 20, 123-134.

(Manuscript received June 16, 2007; revision accepted for publication January 22, 2008.) 